\title{
O IMPACTO DA DOENÇA DE ALZHEIMER NO CUIDADOR
}

\author{
Marília da Nova Cruz \\ Amer Cavalheiro Hamdan\#
}

\begin{abstract}
RESUMO. O crescimento da população idosa é um fenômeno mundial. Em 2025 o Brasil será o $6^{\circ}$ país do mundo quanto ao contingente de idosos. Concomitantemente com o aumento da população idosa ocorre o aumento das doenças crônicodegenerativas, como a Doença de Alzheimer (DA). A DA é caracterizada por um declínio progressivo nas áreas de cognição, função e comportamento. À medida que a doença progride surge a demanda por cuidados especiais, função importante desempenhada pelos cuidadores. Pesquisas têm evidenciado que o declínio do paciente e suas demandas específicas são fatores importantes de estresse para o cuidador. Estes apresentam altos índices de sintomas psiquiátricos - especialmente a depressão -, prejuízos no sistema imunológico, assim como altos índices de conflitos familiares. Outros estudos sugerem que altos índices de sobrecarga e impacto no cuidador estão associados à institucionalização do paciente. O presente estudo de revisão tem por objetivo levantar os principais aspectos determinantes do impacto no cuidador de pacientes com DA.
\end{abstract}

Palavras-chave: doença de Alzheimer, cuidador, envelhecimento.

\section{ALZHEIMER DISEASE: ITS IMPACT ON THE HEALTH CARE NURSE}

\begin{abstract}
Increase in the elderly population is a worldwide phenomenon. In 2025 Brazil will be the sixth country in the world in the number of elderly people. Consequently, there will also be an increase in chronic-degenerative diseases, such as Alzheimer Disease (AD.). $\mathrm{AD}$ is described as a progressive decline in cognition areas, function and behavior. During its progressive development, the $\mathrm{AD}$ person requires special care which is administered by health care nurses. Researches evidenced that the patient's deterioration stage and his/her requirements are important stress factors to the care nurses. These manifest high indexes of psychiatric symptoms, mainly depression, damage at the immunological system and also high familiar disagreement indexes. Other studies suggest that these high indexes and burden on the care nurses are associated with the patient's institutionalization. Current revision study shows the main determinant aspects on the impact on care nurses with AD patients.
\end{abstract}

Key words: Alzheimer's Disease, care nurse, aging.

\section{EL IMPACTO DE LA ENFERMEDAD DE ALZHEIMER EN EL CUIDADOR}

RESUMEN. El crecimiento de la población anciana es un fenómeno mundial. En 2025 Brasil será el $6^{\circ}$ país del mundo cuanto al contingente de ancianos. Concomitantemente con el aumento de la población anciana ocurre el aumento de las enfermedades crónico-degenerativas, como la Enfermedad de Alzheimer (DA). La EA es caracterizada por un declino progresivo en las áreas de cognición, función y comportamiento. A la medida que la enfermedad avanza surge la demanda por cuidados especiales, función importante desempeñada por los cuidadores. Pesquisas han evidenciado que el declino del paciente y sus demandas específicas son factores importantes de estrese para el cuidador. Estos presentan altos índices de síntomas psiquiátricos - especialmente la depresión -, perjuicio en el sistema inmunológico, así como altos índices de conflictos familiares. Otros estudios sugieren que altos índices de sobrecarga e impacto en el cuidador están asociados a la institucionalización del paciente. El presente estudio de revisión tiene por objetivo levantar los principales aspectos determinantes del impacto en el cuidador de pacientes con EA.

Palabras-clave: Enfermedad de Alzheimer; cuidador. envejecimiento.

O crescimento da população idosa é um fenômeno mundial. Em decorrência do envelhecimento populacional, há também um aumento das doenças crônico - degenerativas relacionadas à idade, como as demências (Caramelli \& Barbosa, 2002). A causa mais freqüente de demência é a Doença de Alzheimer

Psicóloga. Departamento de Psicologia, Universidade Federal do Paraná.

* Doutora em Psicologia. Departamento de Psicologia, Universidade Federal do Paraná. 
(DA), representando $50 \%$ a 70\% dos casos (Câmara, Menezes \& Campos, 1998; Caramelli \& Barbosa, 2002). A prevalência da DA após os 65 anos de idade duplica a cada cinco anos (Caramelli \& Barbosa, 2002; Dunkin \& Hanley, 1998).

A DA se caracteriza como um processo neurodegenerativo, progressivo (Caramelli \& Barbosa, 2002; Petrilli, 1997), relacionado à idade e de etiologia incerta. O quadro clínico da DA é caracterizado por alterações comportamentais e cognitivas, incluindo o comprometimento da memória, do pensamento e do raciocínio. O sintoma mais evidente no início da doença é o comprometimento da memória recente (Caramelli \& Barbosa, 2002). A evolução da DA é de caráter descendente e o nível de gravidade é comumente dividido em três estágios: leve, moderado e grave (Abreu, Barros \& Forlenza, 2005; Câmara et al., 1998). Em cada uma das etapas observa-se uma gradativa perda da autonomia e o conseqüente aumento das necessidades de cuidados e supervisão de terceiros para os portadores da doença (Abreu et al. , 2005).

O cuidador desempenha um papel essencial na vida dos pacientes com a DA. Este estudo tem por objetivo levantar os principais aspectos determinantes de impacto no cuidador de pacientes com DA. Através de levantamento bibliográfico realizados nos sistemas Scielo, MedLine e Lilacs foram identificados artigos que estivessem ligados às relações entre DA e o impacto no cuidador.

\section{O CUIDADOR DA DOENCAA DE ALZHEIMER}

\section{Quem é o cuidador}

À medida que a DA progride, surge a demanda por cuidados especiais, função importante desempenhada pelos cuidadores. Aproximadamente $80 \%$ dos cuidados com os pacientes com DA são providos por membros da família (Haley, 1997). O conceito de cuidador vem sendo bastante discutido na literatura, contudo ainda não há consenso sobre sua definição, gerando interferências nas pesquisas sobre o ato de cuidar (Garrido \& Almeida, 1999). Segundo Grafstrom, Fratiglioni, Sandman e Winblad (1992), o cuidador é quem dá suporte físico e psicológico, fornecendo ajuda prática, se necessário. Petrilli (1997) define cuidador como a pessoa diretamente responsável pelos cuidados do paciente - normalmente a esposa, um dos filhos ou outro parente, ou, ainda, uma pessoa contratada para a função. De acordo com Garrido e Almeida (1999), o cuidador é definido como o principal responsável por prover ou coordenar os recursos requeridos pelo paciente. Os estudos sobre cuidadores são recentes na literatura. $\mathrm{Na}$ década de 60 iniciaram-se estudos sobre cuidadores de pacientes psiquiátricos; posteriormente, de cuidadores de idosos frágeis; finalmente, na década de 80 iniciaram-se estudos sobre cuidadores de pessoas com demência, em especial cuidadores de pacientes com DA (Mohide, 1993). A literatura sobre cuidadores em países desenvolvidos é extensa, porém, no Brasil, estudos sobre cuidadores de idosos com demência ainda são escassos (Karsch, 2003).

Pesquisas evidenciam que, em sua maioria, os cuidadores são do sexo feminino, (Burns, Nichols, Adams, Graney \& Lummus, 2003; Dunkin \& Hanley, 1998; Garrido \& Almeida, 1999; Garrido \& Menezes, 2004; Haley, 1997; Hinrichsen \& Niederehe, 1994; Karsch, 2003; Mohide, 1993; Taub, Andreoli \& Bertolucci, 2004), especialmente esposas e filhas do paciente (Haley, 1997; Karsch, 2003; Mohide, 1993). Este fato reflete um padrão cultural em que o papel de cuidador ainda é visto como uma função feminina (Goldfarb \& Lopes, 1996; Karsch, 2003).

O padrão cultural de dependência das crias em relação às mães empurra novamente a mulher do núcleo familiar para o papel de tutora quando um adulto dependente necessite de cuidados (Karsch, 2003). Não obstante, há casos em que os idosos recebem assistência de mais de um cuidador, ou seja, de um cuidador secundário. Destes, 40\% são do sexo masculino (Mohide, 1993).

Pesquisas com cuidadores de idosos demenciados indicam que a idade média do cuidador varia entre 50 e 65 anos (Burns et al., 2003; Garrido \& Almeida, 1999; Garrido \& Menezes, 2004; Hinrichsen \& Niederehe, 1994; Taub et al., 2004), sendo que 33\% têm idade acima de 60 anos (Mohide, 1993).

O cuidador desempenha um papel essencial na vida diária dos pacientes com DA, envolvendo-se em praticamente todos os aspectos do cuidado e assumindo responsabilidades adicionais de maneira crescente (Engelhardt, Dourado \& Lacks, 2005). À medida que a demência vai progredindo, o cuidador, além de se envolver em atividades da vida diária (AVD) instrumentais, como administrar finanças e medicamentos, aumenta suas responsabilidades em AVDs básicas, como tarefas de cuidado pessoal de higiene, banho e alimentação (Haley, 1997). Em média, os cuidadores despedem 60 horas por semana em responsabilidades de cuidador (Haley, 1997; Mohide, 1993). O cuidador geralmente é escolhido dentro do círculo familiar e, muitas vezes, a tarefa é assumida de maneira inesperada, sendo ele conduzido a uma sobrecarga emocional (Taub et al., 2004). 


\section{O impacto da doença no cuidador}

A sobrecarga dos cuidadores é um dos mais importantes problemas causados pela demência (Grafstrom et al., 1992; Taub et al., 2004). O termo Burden (impacto) da língua inglesa tem recebido bastante atenção da literatura e é utilizado para descrever os aspectos negativos associados ao cuidado de um paciente dependente (Dunkin \& Hanley, 1998; Garrido \& Almeida, 1999; Mohide, 1993). Estes aspectos dizem respeito à presença de problemas, dificuldades ou eventos adversos que afetam significativamente a vida dos responsáveis pelo paciente (Garrido \& Almeida, 1999) nos âmbitos físico, financeiro e emocional (Dunkin \& Hanley, 1998).

$\mathrm{Na}$ literatura, estudos com grupos-controle evidenciam algumas diferenças entre cuidadores de idosos demenciados e outros grupos populacionais. Um estudo comparativo entre cuidadores de três grupos geriátricos diferentes - pacientes com DA, pacientes com demência vascular e idosos não demenciados - revelou que o impacto sofrido pelos cuidadores é maior em cuidadores de pacientes com DA (Rainer et al., 2002). Outros estudos indicaram que os cuidadores de pacientes com demência apresentavam maiores índices de impacto que cuidadores de pacientes com depressão, AVC ou idosos hígidos da comunidade (Garrido \& Almeida, 1999). Cuidadores de pacientes com DA possuem maiores chances de ter sintomas psiquiátricos, mais problemas de saúde, maior freqüência de conflitos familiares e problemas no trabalho, se comparados a pessoas da mesma idade que não exercem o papel (Cerqueira \& Oliveira, 2002). Outro estudo evidencia que cuidadores de idosos dementes apresentam um pior julgamento sobre a própria saúde, sentem-se mais estressados e com a vida afetiva e social mais limitada do que cuidadores de idosos não dementes (Grafstrom et al., 1992).

O impacto sofrido pelos cuidadores pode ser observado também na utilização de serviços de saúde, já que cuidadores de pacientes com DA consultam $46 \%$ mais médicos e utilizam mais medicamentos psicotrópicos - como antidepressivos e antipsicóticos do que cuidadores de pacientes que não têm DA (Garrido \& Almeida, 1999; Grafstrom et al., 1992; Haley, 1997). Pesquisas demonstram que os cuidadores apresentam uma piora na saúde física (Garrido \& Menezes, 2004), um prejuízo no sistema imunológico, que pode persistir até quatro anos após o falecimento do paciente (Haley, 1997). Em decorrência do impacto, até $60 \%$ dos cuidadores podem desenvolver sintomas físicos e psicológicos.
Os sintomas físicos mais comuns são: hipertensão arterial, desordens digestivas, doenças respiratórias e propensão a infecções. Sintomas psicológicos freqüentes são: depressão, ansiedade e insônia (Engelhardt et al., 2005). A saúde precária do cuidador é um fator que contribui para a institucionalização do paciente (Dunkin \& Hanley; 1998; Haley, 1997; Grafstrom et al., 1992). Os altos índices de depressão em cuidadores de pacientes com DA são evidenciados com freqüência na literatura (Cerqueira \& Oliveira, 2002; Engelhardt et al., 2005; Garrido \& Almeida, 1999; Garrido \& Menezes, 2004; Grafstrom et al., 1992; Haley, 1997; Rodrigues, Andrade \& Mendes, 1995), chegando a atingir entre 30 e $55 \%$ dos cuidadores, os quais sofrem risco de desenvolver a doença duas a três vezes maior que o restante da população.

Para avaliar o impacto nos cuidadores é essencial que se desenvolvam instrumentos que possam medi-lo (Taub et al., 2004). Para atingir este objetivo, em 1985, Zarit e colaboradores desenvolveram a escala The Zarit Burden Interview (ZBI). A escala possui 22 questões que avaliam o impacto do cuidado nas esferas física, psicológica e social, com uma pontuação que pode variar de 0-4. A escala é bastante utilizada em pesquisas com cuidadores de idosos demenciados (Cerqueira \& Oliveira, 2002; Garrido \& Menezes, 2004; Taub et al., 2004). No Brasil, a confiabilidade da escala foi avaliada por Taub et al. (2004), demonstrando ser de fácil administração e confiável para medir o impacto apresentado pelos cuidadores de pacientes com demência.

\section{Os fatores de Impacto no cuidador}

O primeiro fator a ser considerado é o posicionamento da família diante da doença. Assim como a doença passa por vários estágios, a família passa por diferentes etapas. A princípio, a família não sabe o que está acontecendo diante das manifestações de déficit do paciente, gerando sentimentos de hostilidade e irritação. Do outro lado, o paciente pode perceber as próprias deficiências, correndo o risco de deprimir-se. À medida que a doença vai evoluindo, ocorre a busca por um diagnóstico, porém, nem sempre os familiares o aceitam rapidamente, podendo negá-lo, na tentativa de recuperar a "pessoa de antes". Após a aceitação do diagnóstico, pode haver uma sensação de catástrofe. As famílias reagirão de maneiras diferentes, dependendo das próprias características (Goldfarb \& Lopes, 1996). Podem ocorrer três posicionamentos indesejáveis entre os familiares: superproteção, evasão da realidade e expectativas exageradas com relação ao desempenho 
do paciente (Câmara et al.,1998). Os parentes que fazem evasão da realidade, ou seja, negam a doença, são geralmente os que mais sofrem; já os que assumem a função de cuidador tendem a monopolizar a função, colocando-se na posição de serem os únicos a fazer as coisas e abdicam de qualquer atividade que represente uma satisfação pessoal, acabando "heroicamente estressados" (Goldfarb \& Lopes, 1996). Quando o paciente não reconhece mais seus familiares há um primeiro luto para a família, devido à "morte social do paciente". O segundo luto ocorre com a morte biológica do ente querido (Goldfarb \& Lopes, 1996).

Há situações nas quais a família não tem condições de cuidar do paciente e recorre à ajuda de uma pessoa, profissional ou não, que será remunerada para exercer o papel. Neste contexto entram em cena os cuidadores formais, sujeitos a passar por diversos conflitos com a família do paciente, podendo ser objeto de projeção de culpas e frustrações que não podem ser aceitas na família. Diante desta situação é recomendável que o cuidador formal seja discreto, passe por uma reciclagem de conhecimentos e possa conversar sobre as ansiedades das quais é portador (Goldfarb \& Lopes, 1996).

Uma característica do paciente com DA é a necessidade de supervisão crescente, originando fatores de estresse para o cuidador. Pearlin (1990), citado por Engelhardt et al. (2005) sugere alguns estressores primários para os cuidadores, dividindo-os em subjetivos e objetivos. Os estressores objetivos vão se modificando ao longo da evolução da DA e incluem nível de dependência em AVDs e presença de distúrbios do comportamento. Os estressores subjetivos correspondem ao modo pelo qual os estressores objetivos são experimentados pelo cuidador. Estes dois estressores podem levar a conflitos familiares, problemas econômicos e a uma sensação de aprisionamento no papel de cuidador.

\section{As variáveis do paciente}

No que diz respeito às variáveis do paciente, as dificuldades em AVDs e o declínio cognitivo do paciente são pontos importantes para o impacto no cuidador (Dunkin \& Hanley, 1998; Engelhardt et al.; 2005; Grafstrom et al., 1992; Haley, 1997; Mohide, 1993). Assistir ao declínio e à morte do paciente constitui um dos aspectos mais traumáticos de cuidar (Haley, 1997).

Por outro lado, além de ser influenciado pelos aspectos cognitivos da demência, o cuidador passa a sofrer também a enfluência dos aspectos comportamentais (Garrido \& Almeida, 1999). Não há um consenso na literatura sobre o conceito de distúrbios do comportamento na demência (Garrido \& Almeida, 1999); no entanto, a Internacional Psychogeriatric Association (IPA), em 1996, cunhou o termo sintomas psicológicos e comportamentais da demência (Tamai, 2002), que inclui sintomas como alucinações, delírios, perambulação, agressividade, depressão, alterações noturnas, etc. (Garrido, 1999; Haley, 1997; Petrilli, 1997, Tamai, 2002). No que diz respeito a estes sintomas, há consenso na literatura de que são fatores preditores do impacto no cuidador (Dunkin \& Hanley, 1998; Donaldson, Tarrier \& Burns, 1998; Engelhardt et al., 2005; Garrido \& Almeida, 1999; Garrido \& Menezes, 2004; Grafstrom et al., 1992; Haley, 1997; Hinrichsen \& Niederehe, 1994; Petrilli, 1997; Rainer et al., 2002; Tamai, 2002). Estes fatores são considerados os mais importantes para o impacto, tendo influência maior que a deterioração cognitiva e a dificuldade em AVDs (Donaldson et al., 1998; Garrido \& Menezes, 2004; Engelhardt et al., 2005; Rainer et al., 2002), estando associados à depressão do cuidador (Haley, 1997) e à institucionalização do paciente (Dunkin \& Hanley, 1998). As alterações comportamentais do paciente podem ser uma tentativa de comunicação, e cabe ao cuidador investigar as circunstâncias que estão causando esta alteração de atitude, sendo inútil discutir com o paciente (Petrilli, 1997).

\section{As variáveis do cuidador}

No que tange às variáveis do cuidador, um maior impacto está associado à saúde precária do cuidador (Garrido \& Menezes, 2004; Grafstrom et al., 1992; Hinrichsen \& Niederehe, 1994) e ao grau de parentesco, sendo os cônjuges os que sofrem o maior impacto (Grafstrom et al., 1992; Haley, 1997). Uma hipótese para isto é que, devido à sua idade mais avançada, o cônjuge está mais suscetível a problemas de saúde, fator relacionado a um maior impacto (Dunkin \& Hanley, 1998). Cuidadores mulheres costumam sofrer um impacto maior, possivelmente pelas diferenças de tarefas entre o cuidador do sexo feminino e o do masculino. As mulheres assumem com mais freqüência tarefas desgastantes, como a higiene do paciente, além de terem que gerenciar as tarefas domésticas (Dunkin \& Hanley, 1998). A relação pré-mórbida entre o paciente e o cuidador também é um fator relevante. Cuidadores com uma relação pré-mórbida satisfatória com o paciente apresentam um menor impacto (Cerqueira \& Oliveira, 2002; Dunkin \& Hanley, 1998; Garrido \& Almeida, 1999; Mohide, 1999) e menores chances de depressão (Dunkin \& Hanley, 1998). 
Estudos que avaliavam o impacto nos cuidadores de pacientes com DA revelam que altos índices de emoção expressada (EE) - atitudes de oposição, envolvimento excessivo e hostilidade para com o paciente - estão relacionados a um maior impacto no cuidador, maiores índices de depressão e aumento dos sintomas psicológicos e comportamentais do paciente (Dunkin \& Hanley, 1998; Hinrichsen \& Niederehe, 1994). Hinrichsen e Niederehe (1994) realizaram um estudo no qual um dos objetivos era correlacionar três estratégias de gerenciamento da demência e ajustamento familiar. As estratégias de gerenciamento incluíam: oposição (autoritarismo do cuidador), encorajamento (esforços para fazer com que o paciente veja o lado bom da vida) e gerenciamento ativo (atividades que envolvam a proteção do paciente). A pesquisa concluiu que cuidadores que utilizam a estratégia de oposição sofrem maior impacto, têm mais sintomas psiquiátricos e maior desejo de institucionalização do paciente. Cuidadores que utilizam com mais freqüência estratégias de encorajamento apresentam menor impacto e menor desejo de institucionalização do paciente. Altos índices de gerenciamento ativo estão correlacionados a maior impacto e maior desejo de institucionalização do paciente.

Com relação ao tempo de cuidado, não há consenso na literatura quanto à relação entre impacto e tempo. Um estudo mostrou que o impacto no cuidador tende a melhorar ao longo do tempo (Hinrichsen \& Niederehe, 1994). Outro evidenciou que quanto maior o tempo de cuidado maior é também o impacto no cuidador (Garrido \& Menezes, 2004), possivelmente porque, com o passar do tempo, ocorre uma maior sobrecarga do cuidador (Garrido \& Menezes, 2004; Engelhardt et al., 2005).

Cuidadores que têm um suporte social, ou seja, que se engajam em atividades na comunidade, participam de grupos de apoio e adaptam-se melhor à função de cuidador apresentam um menor impacto (Cerqueira \& Oliveira, 2002; Dunkin \& Hanley, 1998; Garrido \& Almeida, 1999; Haley, 1997) e níveis mais baixos de depressão e maiores de satisfação (Haley, 1997) do que cuidadores que têm pouca disponibilidade de suporte social (Dunkin \& Hanley, 1998; Garrido \& Almeida, 1999; Haley, 1997). Tanto nos Estados Unidos como na Europa há investimentos em políticas públicas a fim de construir e manter redes de suporte a idosos e a cuidadores. Um exemplo disso é a rede de suporte a cuidadores que se define como community care, cujo objetivo é manter o idoso em casa, oferecendo suporte para a família e para o cuidador, trazendo, por exemplo, um profissional para alternar os cuidados com o cuidador (Karsch, 2003).

\section{As estratégias de Intervenção com cuidadores}

Intervenções em cuidadores têm surgido nos últimos 15 anos, porém só recentemente estão sendo estudadas, havendo também uma carência de estudos controlados sobre o assunto (Dunkin \& Hanley, 1998). O objetivo da maioria das intervenções é mudar a forma como o cuidador interage com o paciente em casa. Melhores estratégias de gerenciamento de problemas influenciam no ajustamento emocional do cuidador, refletindo-se em uma melhor assistência por parte do paciente (Hinrichsen \& Niederehe, 1994). Estas intervenções podem ajudar a amenizar a angústia do cuidador, evitar a institucionalização do paciente e permitir que a família possa fazer planos para o futuro (Haley, 1997). A literatura aponta diferentes tipos de intervenção utilizados com cuidadores de idosos demenciados: grupos de apoio, intervenções psicoeducacionais, terapia familiar e terapia individual. A seguir serão relatadas mais detalhadamente essas diferentes formas de intervenção.

Os grupos de apoio se caracterizam por um espaço de troca de informações entre cuidadores (Haley, 1997), e seus benefícios incluem educação e suporte social (Dunkin \& Hanley, 1998). Há também um efeito terapêutico resultante da identificação entre as pessoas que compartilham de um mesmo problema. Os participantes do grupo levam apoio e esclarecimentos sobre a doença (Goldfarb \& Lopes, 1996). Alguns estudos com grupos-controle relataram redução de sintomas psiquiátricos no cuidador, porém não observeram redução no impacto. Os grupos de apoio são indicados para cuidadores que possuem um baixo nível de impacto, não sendo apropriados como único recurso a cuidadores com alto nível de impacto (Dunkin \& Hanley, 1998).

A terapia familiar é indicada para famílias com questões mal-resolvidas que interferem no cuidado do paciente; entretanto, ainda não existem estudos sistematizados que comprovem a sua eficácia (Dunkin \& Hanley, 1998).

A terapia individual é indicada para cuidadores com psicopatologia, com um alto nível de impacto (Dunkin \& Hanley, 1998), e quando a relação prémórbida paciente-cuidador é insatisfatória (Goldfarb \& Lopes, 1996). Estas intervenções, segundo estudos controlados, apresentam melhores resultados sobre o impacto e depressão do cuidador do que intervenções grupais (Dunkin \& Hanley, 1998). 
As intervenções psicoeducacionais vão além da simples transmissão de informações técnicas, podendo ser vistas como o estabelecimento de um fluxo de informações de terapeuta para paciente e vice-versa, na tentativa de implementar, nos familiares e profissionais, recursos para lidar com a doença. Podem ser aplicadas individualmente, em família ou em diversos tipos de grupo (Justo \& Calil, 2004). As intervenções psicoeducacionais podem beneficiar todos os tipos de cuidadores, mesmo em uma única sessão com o médico (Dunkin \& Hanley, 1998). Sessões psicoeducacionais informam sobre particularidades da doença, sintomas típicos e curso esperado (Dunkin \& Hanley, 1998). Estas intervenções de caráter estruturado focam habilidades específicas dos cuidadores, auxiliam na administração dos distúrbios do comportamento do paciente e incentivam os cuidadores a fazer atividades prazerosas (Haley, 1997). As intervenções psicoeducacionais em cuidadores podem beneficiá-los através de uma redução do impacto (Hinrichsen \& Niederehe, 1994) resultante da aprendizagem de estratégias de gerenciamento de sintomas psicológicos e comportamentais (Dunkin \& Hanley, 1998; Hinrichsen \& Niederehe, 1994) e do desenvolvimento de habilidades de comunicação com o paciente (Dunkin \& Hanley, 1998).

\section{CONCLUSÃO}

Os problemas dos cuidadores constituem um dos aspectos mais importantes da DA (Haley, 1997). Neste sentido, a avaliação dos cuidadores deveria fazer parte do tratamento da demência (Dunkin \& Hanley, 1998), uma vez que o suporte aos familiares e cuidadores é de extrema importância (Garrido \& Menezes, 2004; Petrilli, 1997). São também necessários a implantação e ampliação de intervenções efetivas (Cerqueira \& Oliveira, 2002; Garrido \& Menezes, 2004), uma boa formação de profissionais da saúde especializados nessa área e investimentos em pesquisas no âmbito da prevenção do impacto no cuidador (Garrido \& Menezes, 2004). A atenção aos cuidadores de pacientes demenciados é essencial, pois se reflete em uma melhor qualidade de vida não só para o cuidador, mas principalmente para o paciente. "A melhor ajuda para o paciente é ajudar o cuidador" (Fala de um cuidador, Burns et al., 2003).

\section{REFERÊNCIAS}

Abreu, I. D, Barros, H. L \& Forlenza, O. V. (2005). Demência de Alzheimer: correlação entre memória e autonomia. Revista de Psiquiatria Clínica, 32(3), 131-136.
Burns, M. D, Nichols, L. O, Adams, J. M, Graney, M. J \& Lummus, A. (2003). Primary Care Interventions for Dementia Caregivers: 2- Year Outcomes From the REACH Study. The Gerontologist, 43(4), 547-555.

Câmara, D. C, Menezes, A. K. \& Campos, C. M. T. (1998). Atendimento interdisciplinar a pacientes com demência e seus cuidadores. Arquivos de Geriatria e Gerontologia, 2(1), 11-21.

Caramelli, P. \& Barbosa, M. T. (2002). Como diagnosticar as quatro causas mais freqüentes de demência?. Revista Brasileira de Psiquiatria, 24(Supl I), 7-10.

Cerqueira, A. T. A. R. \& Oliveira, N. I. L. (2002). Programa de Apoio a cuidadores: uma ação terapêutica e preventiva na atenção à saúde dos idosos. Psicologia USP, 13(1), $133-150$.

Dunkin, J. J. \& Hanley, C. A. (1998). Dementia caregiver burden: A review of the literature and guidelines for assessment and intervention. Neurology, 51(Supl. 1), 5360.

Donaldson, C., Tarrier, N. \& Burns, A. (1998). Determinats of carer stress in Alzheimer's disease. International Journal of Geriatric Psychiatry, 13(4), 248-256.

Engelhardt, E., Dourado, M. \& Lacks, J. (2005). A Doença de Alzheimer e o impacto nos cuidadores. Revista Brasileira de Neurologia, 14(2), 5-11.

Garrido, R. \& Almeida, O. P. (1999). Distúrbios de comportamento em pacientes com demência: impacto na vida do cuidador. Arquivos de Neuropsiquiatria, 57(2B), 427-434.

Garrido, R. \& Menezes, P. R. (2004). Impacto em cuidadores de idosos com demência atendidos em um serviço psicogeriátrico. Revista de Saúde Pública, 38(6), 835841.

Goldfarb, D. C. \& Lopes, R. G. C. (1996). A família frente à situação de Alzheimer. Gerontologia, 4(1), 33-37.

Grafstrom, M., Fratiglioni, L., Sandman, P. O. \& Winblad, B. (1992). Health and social consequences for relatives of demented and non-demented elderly: A population study. Journal Clinical of Epidemiology, 45(8), 861-870.

Haley, W. (1997). The family caregiver's role in Alzheimer's disease. Neurology, 48(5), 25-29.

Hinrichsen, G. A. \& Niederehe, G. (1994). Dementia Management Strategies and Adjustment of Family Members of older patients. The Gerontologist, 34(1), 95102.

Justo, L. P. \& Calil, H. M. (2004). Intervenções Psicossociais no transtorno bipolar. Revista de Psiquiatria Clínica, 31(2), 91-99.

Karsch, U. M. (2003). Idosos dependentes: famílias e cuidadores. Caderno de Saúde Pública, 19(3), 861-866.

Mohide, E. A. (1993). Informal care of community- dwelling patients with Alzheimer's disease: Focus on the family caregiver. Neurology, 43(Supl. 4), 16-19.

Petrilli, L. A. G. C. (1997). Orientação da família do doente de Alzheimer: pontos de consenso. Sobre Comportamento e Cognição, 3, 216-225. 
Rainer, M., Jungwisth, S., Kruger-Rainer, C., Croy, A., Gatterir, G. \& Hausshofer, M. (2002). Pflegende Angehorige von Demenzerkrankten: Belastungsfaktaren und deren Auswirkung. Psychiatrische Praxis, 29(3), 142-147.

Rodrigues, R. A. P., Andrade, O. G. \& Mendes, M. M. R. (1995). Estratégias para o Cuidado Domiciliar de Idosos com a Doença de Alzheimer. Gerontologia, 3(2), 73-80.

Smith, M. A. C. (1999). Doença de Alzheimer. Revista Brasileira de Psiquiatria, 21, 3-7.
Taub, A., Andreoli, S. B. \& Bertolucci, P. H. (2004). Dementia caregiver burden: Reliability of the Brazilian version of the Zarit caregiver burden interview. Caderno de Saúde Pública, 20(2), 372-376.

Recebido em 19/09/2006 Aceito em 30/11/2006

Endereço para correspondência:

Marília da Nova Cruz. Bruno Filgueira, 73, apt 81, Bairro Água Verde, CEP 80240220 Curitiba-PR.E-mail: marilia_danova@yahoo.com.br 